\title{
Zum sprachlichen Ausdruck der Simultaneität. Eine Untersuchung zum Deutschen
}

\author{
Temporal relations of simultaneity in the German language \\ Mariola WIERZBICKA ${ }^{1}$ \\ Uniwersytet Rzeszowski
}

\begin{abstract}
The paper discusses ways of expressing temporal relations of simultaneity in the German language. Although the relations can be expressed by participle phrases, noun phrases, prepositional phrases and infinitive phrases, the adverbial clause is the most frequent means of expressing the relations. The temporal adverbial clause has an almost unlimited range of application, which stretches from a vaguely hinted relation to absolute necessity, and from general statements and clarifications to definite emotionally motivated utterances. Wherever there is an obvious connection between facts, events, actions, relations as well as personal will and feeling, it can be expressed by means of a temporal structure. The subject of the paper is the influence of conjunctions während, als, wenn, seit(dem) and solange on the time arrangement of situations introduced into the time clause and the main clause with regard to morphological, syntactic and semantic elements and dependence on the relation of the correspondence between events in the time clause and events in the main clause in German. Keywords: temporal relation, temporal clauses, simultaneity
\end{abstract}

1 https://orcid.org/0000-0003-2125-0204

Uniwersytet Rzeszowski

Instytut Neofilologii

mwierzb@wp.pl 


\section{Zusammenfassung}

Der vorliegende Beitrag diskutiert den sprachlichen Ausdruck von Relationen der völligen Gleichzeitigkeit im Deutschen. Obwohl es zur Bezeichnung temporaler Beziehungen eine Liste von Möglichkeiten gibt, wie Partizipial-, Nominal-, Präpositional- oder Infinitivphrasen, ist das eigentliche Temporalsatzgefüge das bei weitem häufigste temporale Ausdrucksmittel. Das Temporalsatzgefüge hat nahezu unbeschränkten Anwendungsbereich, der von dem fast nur angedeuteten Zusammenhang bis zur zwingenden Notwendigkeit, von Allgemeinaussagen und didaktischen Erläuterungen bis zu emotional bestimmten Motivationen reicht. Wo immer ein temporaler Zusammenhang zwischen Tatsachen, Ereignissen, Handlungen und Verhältnissen oder auch zwischen persönlichem Wollen und Fühlen gesehen wird, lässt sich dies in einem Temporalgefüge darstellen. Den Untersuchungsgegenstand dieses Beitrages bildet darüber hinaus der Einfluss von den Subjunktoren während, als, wenn, seit(dem) und solange auf die zeitliche Anordnung der im Temporalsatz und im übergeordneten Satz eingeführten Situationen unter Berücksichtigung verschiedener morphologischer, syntaktischer und semantischer Faktoren.

Schlüsselwörter: temporale Relationen, Temporalsatzgefüge, völlige Gleichzeitigkeit

\section{Einleitende Bemerkungen}

Allgemein gilt, dass in komplexen Sätzen, die aus je mindestens zwei satzartigen Konstruktionen bestehen, zwei Sachverhalte zueinander in Beziehung gesetzt werden. Je nach der kategorialen Zuordnung der Teilsätze handelt es sich um unterschiedliche Beziehungen. Immer aber spielt die zeitliche Beziehung zwischen den Teilsätzen eine Rolle. Diese drückt sich auch in der Wahl der Tempora aus. Grundsätzlich erfolgt die Wahl der Tempora, die sowohl für ein bestimmtes Tempus in einem singulären Satz als auch für die Tempora in einem fortlaufenden Text gilt, im Kommunikationsakt jeweils aufgrund der Intention des Sprechers. Die Tempuswahl kann dabei Restriktionen stilistischen Charakters unterliegen oder darüber hinaus von anderen Faktoren abhängen, die die jeweilige Tempuswahl entweder gebieten oder aber verbieten. Als ein solcher Faktor, auf den mit der Lehre von der consecutio temporum Bezug genommen wird, kann das komplexe Satzgefüge angesehen werden. Von Bedeutung ist dabei die Frage, ob und wieweit gegebenenfalls die Wahl eines entsprechenden Tempus im Nebensatz vom Nebensatz-Typ als solchem abhängt. Bei den zeitlichen Verhältnissen unterscheidet man Gleichzeitigkeit, Vorzeitigkeit und Nachzeitigkeit der Sachverhalte in einem übergeordneten Satz und in einem Temporalsatz. 


\section{Gleichzeitigkeitsrelationen}

Verfügen zwei Sachverhalte über annähernd dieselbe Dauer, geht man davon aus, dass es sich um ein Temporalsatzgefüge der völligen Gleichzeitigkeit handelt. Decken sich die Sachverhalte beider Teilsätze in ihrer gesamten Dauer bzw. zeitlichen Erstreckung vollkommen, so werden sie in diesen Temporalsatzgefügen als völlig gleichzeitig dargestellt. Dabei kann das zeitliche Ausmaß unterschiedlich lang sein, d. h. von einer kurzen Länge bis hin zu einer längeren Dauer. Je nachdem durch welchen Subjunktor ein Temporalsatz eingeleitet wird und je nachdem wie die Teilsatzverben dargestellt werden, d. h. in ihrer Dauer bzw. Nicht-Dauer, oder aber als abgeschlossen bzw. nichtabgeschlossen, kann zudem zwischen einer völligen Gleichzeitigkeit und zeitlich komprimierten Akten differenziert werden ${ }^{2}$. Bei den Temporalsatzgefügen der völligen Gleichzeitigkeit, deren Temporalsatz durch die Subjunktoren während, als, wenn, seit(dem) und solange eingeleitet wird, können die Sachverhalte in beiden Teilsätzen in ihrer simultanen Dauer nicht nur ohne Angabe ihres Anfangs- oder Endpunktes, sondern auch in ihrem Verlauf dargestellt werden. Dabei wird der Sachverhalt im Temporalsatz immer, der Sachverhalt im übergeordneten Satz jedoch meistens in seinem Nicht-Abschluss angegeben.

Die Gleichzeitigkeit der Sachverhalte in beiden Teilsätzen wird durch die den Temporalsatz einleitenden Subjunktoren während, als, wenn, seit(dem) und solange ausgedrückt, die die zeitlichen Aspekte der Gleichzeitigkeit kennzeichnen.

$$
\begin{aligned}
& \text { Übergeordneter Satz }--\frac{}{\text { Temporalsatz }} \quad-- \text { während, als ... - - - }
\end{aligned}
$$

Dabei wird der Subjunktor als zur Kennzeichnung eines einmaligen Auftretens eines Sachverhalts oder Zustands in der Vergangenheit verwendet, der Subjunktor wenn dazu, einen einmaligen Sachverhalt in Gegenwart und Zukunft $\mathrm{zu}$ kennzeichnen. Innerhalb der Temporalsatzgefüge der Gleichzeitigkeit sind sowohl formal-strukturell als auch semantischfunktional verschiedene Gefüge-Arten vorzufinden. Der gleichzeitig zum Sachverhalt des übergeordneten Satzes stattfindende Sachverhalt eines

2 Pott (1984:114) schlägt für diese beiden Arten der Gleichzeitigkeit "Kongruenz" und "Identität" vor. Besser und verständlicher ist es, hier von durativer und punktueller Gleichzeitigkeit zu sprechen. 
Temporalsatzes antwortet auf die Frage wann? bei den Subjunktoren wie während, als, und wenn; auf seit wann? beim Subjunktor seit(dem) und auf wie lange? beim Subjunktor solange. Der Inhalt eines Temporalsatzes kann weiter differenziert werden, je nachdem, ob es sich um völlige Gleichzeitigkeit handelt, die anzeigt, dass der Sachverhalt im Temporalsatz simultan bzw. komprimiert gleichzeitig zum Sachverhalt im übergeordneten Satz stattfindet oder um eine partielle Gleichzeitigkeit des Sachverhalts im Temporalsatz. Die Grenzen zwischen diesen beiden Temporalsatzgefügen sind jedoch, wie sich in den einzelnen Analysen zeigen wird, nicht immer eindeutig zu ziehen.

\section{Untersuchungen zur völligen Gleichzeitigkeit}

In einem Temporalsatzgefüge der völligen Gleichzeitigkeit überlappt sich der im übergeordneten Satz mitgeteilte Sachverhalt mit dem im Temporalsatz beschriebenen Sachverhalt.

In einem während-Temporalsatz können nur solche Sachverhalte erscheinen, die beidseitig begrenzt oder aber begrenzbar sind, d.h. nur solche, die über einen definiten Anfang und ein definites Ende verfügen, und bei denen davor und danach je eine gegenteilige Zustandsphase vorliegt. Diese Beschränkung erklärt auch, warum in während-Temporalsatzgefügen solche Zustände wie Kind/jung/alt sein nicht zugelassen sind (1). Die Tatsache, dass für den Zustand des Jungseins einer Person keine früher liegende gegenteilige Phase, in der die Person noch nicht jung war, existiert, da die Person zuvor schlichtweg noch nicht existiert hat, macht die geringe Akzeptabilität von (1) deutlich. Eine analoge Situation liegt für den Zustand des Altseins vor. Demzufolge sind jung bzw. alt sein nur einseitig begrenzte Zustände, da jung sein nur ein Ende, jedoch keinen Anfang mitteilt, während alt sein zwar einen Beginn, aber kein Ende aufweist.

(1) Während ich Kind war, bin ich gerne auf Bäume geklettert (M.W.).

Die Sachverhalte eines während-Temporalsatzes sind meist auf eine konkrete Situation bezogen und dauern in der Regel minimal bzw. normal lange an, d.h. Sekunden, Minuten oder Stunden. Demzufolge bevorzugen länger andauernde Sachverhalte, die sich über einen Zeitraum von mehreren Tagen, Wochen, Monaten oder Jahren erstrecken, wie wohnen oder verreist sein, eher ein als/wenn-Temporalsatzgefüge (2).

(2) Damals, als er bei uns wohnte, war er in sie verliebt (M.W.). 
Das aktuelle Fortschreiten kann durch Temporaladverbien wie gerade oder durch Syntagmen wie dabei sein etwas zu tun hervorgehoben werden, sofern die Sachverhalte im Verlauf ihrer Ausführung dargestellt werden (3).

(3) Während ich dastand und sie ansah, drehte sie allmählich ihr Gesicht aus der Sonne, hin zu mir, und dabei kniff sie das rechte Auge auf eine ulkige Art zusammen. (SRR; 79)

In den während-Temporalsatzgefügen der völligen Gleichzeitigkeit kann der übergeordnete Satz wiederholte Sachverhalte enthalten, die in der Summe ihrer Wiederholungen gleichzeitig zum Sachverhalt im Temporalsatz ausgeführt werden müssen (4).

(4) Ich saß da neben Marie und rauchte vor Aufregung, durch die Disharmonien im Hintergrund vollkommen aus dem Gleichgewicht gebracht, eine Zigarette nach der anderen, während Sommerwind mit Marie plauderte, immer dieses „verzeihende und großzügige Lächeln“ auf dem Gesicht. (BHA; 87)

In der Zeitspanne, die vom während-Temporalsatz angegeben wird, können auch mehrere einzelne Sachverhalte nacheinander realisiert werden (5).

(5) Während er sich umzog, den schicken Anzug und das passende Hemd auswählte und vorm Spiegel prüfte, ob die Krawatte saß, fluchte er in einem fort. (M.W.)

Liegen sowohl das Temporalsatzgeschehen als auch das Geschehen im übergeordneten Satz vor einem zeitlichen Referenzpunkt in der Vergangenheit, d. h. also wenn die sog. Vorvergangenheit mitgeteilt werden soll, erscheint mitunter in beiden Satzteilen Präteritumperfekt (6), meistens kommt Präteritumperfekt jedoch nur im übergeordneten Satz, während im Temporalsatz bei Vorvergangenheit gewöhnlich Präteritum vorkommt (7).

(6) Er hatte hier gestanden, unabänderlich und ehrwürdig, während sie sich daran als an einen alten, vergessenswerten Traum erinnert hatte! (MTB; 130)

(7) Später [...] bestand [sie M.W.] aber darauf, das aus eigener Tasche zu bezahlen, obwohl einer der jungen Beamten, der am Morgen ihre Badezimmertüre bewacht hatte, während sie sich anzog, bereit war, ihr "einen auszugeben". (BHV; 25)

$\mathrm{Da}$ die simultan verlaufenden Sachverhalte in den als/wennTemporalsatzgefügen der völligen Gleichzeitigkeit in ihrer Dauer gesehen werden, erscheint bei Vergangenheitsbezug Präteritum in beiden Teilsätzen. 
Im Gegensatz zu den während-Temporalsatzgefügen werden die Sachverhalte in den als/wenn-Temporalsatzgefügen selten in ihrem Vollzug dargestellt. Demzufolge bezeichnet das Präteritum im Temporalsatz eine meist längere Lebens- bzw. Tätigkeitsperiode, innerhalb welcher sich der Sachverhalt im übergeordneten Satz vollzieht bzw. zu der dieser Sachverhalt gilt (8).

(8) Ich verstand erst später, als ich in Hessen wohnte, wie das gemeint war. (ZGC; 223)

Mitunter kommen neben dem Präteritum auch Präsensperfektgrammeme in den als-Temporalsatzgefügen vor allem in der gesprochenen Sprache vor. Dabei erscheint Präsensperfekt relativ häufig im übergeordneten Satz in Verbindung mit einem Präteritum im Temporalsatz (9). Darüber hinaus sind in den Korpora neben den Kombinationen mit Präteritum in beiden Teilsätzen (10) auch Kombinationen mit Präsensperfekt im übergeordneten Satz (11) belegt.

(9) Als er sich vor etwa sieben Jahren selbständig machte, hat wohl nur er damit gerechnet, daß er so schnell ins große Geschäft kommen würde. (SVD; 9)

(10) Als Ibrahim sich auf den Stufen der verfallenen Fontäne des innersten Hofes niederließ, spürte er seine Müdigkeit. (FLJ; 12)

(11) Noch vorigen Monat, als die Möbelfirma ihr die neuen Sessel gebracht hat, die hier... da hat sie mir, bloß weil ich aufgeschlossen habe, einen Fuffziger spendiert - 'n ganzen Fünfzigmarkschein! (MHR; 11)

Bei kontextbezogener sog. Vorvergangenheit von als-Temporalsatzgefügen erscheint das Präteritumperfekt im übergeordneten Satz in Verbindung mit einem Präteritumperfekt im Temporalsatz (12), ein Präteritumperfekt im übergeordneten Satz und ein Präteritum im Temporalsatz (13) bzw. ein Präteritum im übergeordneten Satz in Verbindung mit einem Präteritumperfekt im Temporalsatz (14).

(12) Dieser Eukalyptus. Den angeblich ihre Eltern gepflanzt hatten, als sie hier als Hippies gezeltet beziehungsweise geschlafsackt hatten. (RIS; 33)

(13) Kein einziger der vier Blaumänner hatte sich auch nur im geringsten erschrocken, als sie mich in die Halle treten sahen. (SVD; 7)

(14) Als ich die Straße wieder erreicht hatte, verlor mein Vorderrad die Luft. (SVD; 51)

In den als/wenn-Temporalsatzgefügen der völligen Gleichzeitigkeit, die als zeitlich komprimierte Akte aufgefasst werden und in denen die Sachverhalte in 
beiden Teilsätzen in ihrem Abschluss dargestellt werden, erscheinen bei Vergangenheitsbezug Präteritum in Aoristbedeutung (15). ${ }^{3}$

(15) Gregor erschrak, als er seine antwortende Stimme hörte, die wohl unverkennbar seine frühere war, in die sich aber, wie von unten her, ein nicht zu unterdrückendes, schmerzliches Piepsen mischte, das die Worte förmlich nur im ersten Augenblick in ihrer Deutlichkeit beließ, um sie im Nachklang derart zu zerstören, daß man nicht wußte, ob man recht gehört hatte. (KFV; 59)

Darüber hinaus können bei den Temporalsatzgefügen der völligen Gleichzeitigkeit, deren Temporalsatz durch die Subjunktoren als/wenn eingeleitet wird, die Sachverhalte in beiden Teilsätzen unabhängig von ihrer tatsächlichen Dauer als zeitlich komprimierte Akte, d.h. als Handlungen von kurzer Dauer und als bereits abgeschlossene dargestellt werden. Wenn in beiden Teilsätzen kurzzeitige Sachverhalte mitgeteilt werden, dann lässt sich nicht eindeutig entscheiden, ob es sich bei diesen Temporalsatzgefügen um eine völlige Gleichzeitigkeit der komprimierten Akte oder aber um die einer Vorzeitigkeit handelt (16).

(16) Als Ellinor vom Pferde stieg, als Leonor ihr die Hand küßte, als die alten Lippen der Mutter ihre eigenen jungen berührten, spürte sie leibhaft die tiefe Gemeinschaft. (FLJ; 301)

Obwohl in den Temporalsatzgefügen der völligen Gleichzeitigkeit komprimierter Akte meistens kurzzeitige Sachverhalte erscheinen, sind auch 'relativ' durative Sachverhalte, die durch die Verwendung der entsprechenden Tempora als zeitlich komprimiert oder aber als punktuell sich vollziehende Akte dargestellt werden, nicht ausgeschlossen. Dabei wird in den meisten Fällen die Zeitspanne, die durch den Sachverhalt im Temporalsatz angegeben wird, zusätzlich durch Temporaladverbiale limitiert (17).

(17) Als Richis in den Stall gekommen war, um ihn aufzusuchen, hatte er sich nur schlafend gestellt, um den Eindruck von Harmlosigkeit, den er an und für sich schon wegen seines Unauffälligkeitsgeruchs ausstrahlte, noch augenscheinlicher zu machen. (SPP; 273)

Bei den seit(dem)-Temporalsatzgefügen der völligen Gleichzeitigkeit wird der Beginn der Zeitspanne, die für die Ausführung des Sachverhalts im übergeordneten Satz relevant ist, durch den Beginn des in der Regel durativen

3 In der Aoristbedeutung werden Sachverhalte in ihrem Abschluß und in der Imperfektbedeutung in ihrer Dauer dargestellt (vgl. dazu Wierzbicka 2004:80, 91). 
Sachverhalts im Temporalsatz mitgeteilt. In den seit-Temporalsatzgefügen wird durch den Temporalsatz der Anfang einer Zeitspanne festgelegt, die bis zur Betrachtzeit andauert und in der der Sachverhalt im übergeordneten Satz abläuft.

Aufgrund ihrer Eigensemantik erscheinen seit-Temporalsatzgefüge immer als zeitlich lokalisiert, d.h. die Sachverhalte in beiden Teilsätzen können kombiniert nicht als wiederholte Sachverhalte auf der Zeitachse vorkommen ${ }^{4}$. Dies hat jedoch seine Gültigkeit für die Fälle, in denen das seitTemporalsatzgefüge als Ganzes gesehen wird. Wie sich im Folgenden zeigen wird, können diese Temporalsatzgefüge durchaus als wiederholt erscheinen, sofern nur ein Einzelsachverhalt entweder im Temporalsatz oder im übergeordneten Satz als wiederholt aufgefasst wird (nach Wierzbicka 2004:99104, Wierzbicka, Schlegel 2008:85f.).

Da der Zeitpunkt, der durch den Sachverhalt im Temporalsatz angegeben wird, immer vor der Sprechzeit liegt und somit vergangen ist, erscheinen die seit-Temporalsatzgefüge nicht mit Zukunftsbezug. Auch solche Sachverhalte, die von der Betrachtzeit in der Vergangenheit aus gesehen zukünftig sind, können in diesen Temporalsatzgefügen nicht vorkommen. Somit sind würde + Infinitiv I bzw. II oder Futur bzw. Futurperfekt von vornherein ausgeschlossen. Wenn ein Futurgrammem im übergeordneten Satz erscheint, so weist das ganze seit-Temporalsatzgefüge keinen Zukunfts-, sondern einen Gegenwartsbezug auf (18). Bei Gegenwartsbezug fällt die Betrachtzeit mit der Sprechzeit zusammen und bei Vergangenheitsbezug liegt eine Betrachtzeit in der Vergangenheit vor.

(18) Es wird ihr wohl gut gehen, seit sie wieder vom Urlaub zurück ist. $=>$ Es geht ihr wohl gut.

Bezüglich der Tempuskombinationen in den seit-Temporalsatzgefügen der völligen Gleichzeitigkeit stimmen die Tempusgrammeme in beiden Teilsätzen meist dann überein, wenn sowohl der Sachverhalt im Temporalsatz als auch der Sachverhalt im übergeordneten Satz die Zeitspanne, die vom Temporalsatz beschrieben wird, vollständig ausfüllen und wenn beide Sachverhalte darüber hinaus gleichzeitig beginnen und bis zur Betrachtzeit parallel verlaufen. Demzufolge erscheint bei Gegenwartsbezug Präsens im übergeordneten Satz, das sich ebenfalls mit Präsens im Temporalsatz verbindet (19).

\footnotetext{
${ }^{4}$ Neumann (1972:141) und Zschunke (1970:222, 1973:99) postulieren: "Das hängt mit der Einmaligkeit der Erzählebene oder des Sprechzeitpunkts zusammen. Der Zeitraum von einem gewissen Punkt in der Vergangenheit bis 'jetzt' läßt sich nicht wiederholen“.
} 
Bei Vergangenheitsbezug erscheint in der Regel in beiden Teilsätzen Präteritum in Imperfektbedeutung (20).

(19) Seit der modelinke Yuppie Jeremias Storck mit der »Ich-möchtegern-ein- großer-Journalist- sein«-Mentalität das >Metropol-Magazin< macht, ist das Blatt eigentlich nur noch als Klolektüre zu verkraften. (SVD; 68)

(20) Ungeachtet der glühenden Mittagshitze traf Adaza schon wenige Minuten nach diesem Coup erste Vorbereitungen und sah sein großes Ziel plötzlich zum Greifen nahe - seit er eine Dunkelkammer besaß und die Wirkungen der Entwicklungsbäder kannte, träumte er davon, mit dem offiziellen Präsidentenporträt, das inklusive der First Lady ja ein Doppelporträt wäre, beauftragt zu werden. (KBI; 177)

Äußert selten treten Sachverhalte in beiden Teilsätzen unabhängig voneinander wiederholt auf, wobei der Sachverhalt im übergeordneten Satz durativ ist und die gesamte Zeitspanne ausfüllt, während die sich wiederholenden Sachverhalte im Temporalsatz in ihrer Summe ein Intervall bilden, dessen Anfang den Ausgangspunkt für den Sachverhalt im übergeordneten Satz darstellt. In (21), in dem beide Teilsatzsachverhalte wiederholt erscheinen, wird durch den nicht-lokalisierten Sachverhalt im Temporalsatz die Zeitspanne angegeben, innerhalb derer der Sachverhalt im übergeordneten Satz sich wiederholend stattfindet.

(21) Und so hören wir am Festtag der Franckeschen Stiftungen, als deren Direktor Paul Raabe am 28. September Abschied nimmt, von ihm auch oft die Sätze, wie wir sie, seitdem die Totschläger im Osten die Straßen immer wieder beherrschen, selten hören: „Es war die Rückgewinnung der kulturellen Einheit, die mich faszinierte. Hier in der Mitte Deutschlands stand die Wiege deutscher Kultur". (ZEIT 40/00; 45)

Bei den seit-Temporalsatzgefügen der völligen Gleichzeitigkeit können die Tempusgrammeme im Temporalsatz und im übergeordneten Satz auch verschieden sein. Im Temporalsatz erscheint in der Regel Präsens bzw. Präteritum in Imperfektbedeutung. Im übergeordneten Satz dagegen kommen bei Gegenwartsbezug neben Präsens und Präsensperfekt selten auch Präteritum und bei Vergangenheitsbezug neben Präsensperfekt, Präteritum in Imperfektbedeutung, Präteritumperfekt und Präteritum in Aoristbedeutung vor. Trotz der Nicht-Übereinstimmung der Tempora im Temporal- und im übergeordneten Satz handelt es sich auch hier um eine völlige Gleichzeitigkeit der Zeitspannen. 
Durch die Verwendung von Präsensperfekt bzw. Präteritumperfekt im übergeordneten Satz bei negierten Sachverhalten, die durch Negationswörter wie nicht/kein mehr, nie(mals), noch nicht, oder einfaches nicht zum Ausdruck gebracht werden, wird mitgeteilt, dass in der relevanten Zeitspanne, die vom Beginn des Sachverhalts im Temporalsatz bis zur Betrachtzeit reicht, der Sachverhalt im übergeordneten Satz, durch ein nicht-negiertes Verb ausgedrückt, noch nicht ausgeführt wurde. Die Verwendung von Präsensperfekt, selten Präteritumperfekt, wird aber dann notwendig, wenn nicht einmal zusätzlich lexikalisch in der Bedeutung kein einziges Mal realisiert wird, oder aber noch als zusätzlicher Teil der Verneinung im übergeordneten Satz vorkommt (22).

(22) Stell dir vor: seit ich wieder vom Urlaub zu Hause bin, habe ich noch kein einziges Mal mein Telefon benutzt. (M.W.)

Darüber hinaus treten Präsensperfekt bzw. Präteritumperfekt im übergeordneten Satz auch bei wiederholten Sachverhalten auf, deren mehrmalige Realisierung durch Temporaladverbiale wie manchmal, oft, immer wieder zusätzlich hervorgehoben werden kann (23).

(23) Seitdem sie in der Stadt war, seit fünf oder sechs Jahren, hatte Katharina sich immer wieder über die nicht vorhandenen Möglichkeiten, „mal einfach irgendwo tanzen zu gehen“, beklagt. (BHV; 14)

Dabei wird Präsensperfekt bzw. Präteritumperfekt dann obligatorisch verwendet, wenn die genaue Zahl der Wiederholungen, die von Beginn des Sachverhalts im Temporalsatz bis zur Betrachtzeit stattgefunden haben, auch mithilfe von Iterationsadverbialen angegeben wird (24).

(24) Es waren fast zweihundert Jahre verflossen, seit die Spinne im Loche gefangen saß, da war ein schlau und kräftig Weib hier Meister, sie war keine Lindauerin, aber doch glich sie Christine in vielen Stücken. (GJS; 42)

Außerdem verbinden sich Präsensperfekt bzw. Präteritumperfekt im übergeordneten Satz mit solchen Sachverhalten, die eine fortschreitende und allmähliche Zustandsveränderung zum Ausdruck bringen. Neben verändern bzw. ändern wird vor allem mithilfe von Verben wie sinken, steigen, wachsen, oder zurückgehen eine quantitative Veränderung mitgeteilt (25). Ähnliches gilt für Sachverhalte, die eine allmähliche Bewusstseinsänderung wie etwas nach und nach entdecken, sich einer Sache bewusst werden anzeigen (26), oder aber beim Auxiliar werden in Verbindung mit meist graduierten Adjektiven (27). 
(25) Wie wir Katharina zu Dank verpflichtet sind: seit sie ruhig und freundlich, auch planvoll unseren Haushalt leitet, sind nicht nur unsere Unkosten erheblich gesunken, sie hat uns auch beide für unsere berufliche Arbeit so frei gemacht, daß wir es kaum in Geld ausdrücken können. (BHV; 35)

(26) Seit ich mich so viel mit ihm befaßte, waren mir seine Probleme bewusst geworden und er war ruhiger geworden und schien nicht dauernd $\mathrm{zu}$ befürchten, ich könnte mich, sobald er fünf Minuten wegging, in Luft auflösen. (HMW; 217)

(27) Meine Tante ist ein bißchen schrullig geworden, seit sie Witwe ist. (SVD; 46)

Durch das Präsensperfekt bzw. Präteritumperfekt wird zum einen mitgeteilt, dass sich der Veränderungsprozess in der Zeitspanne, die durch den Beginn des seit-Sachverhalts im Temporalsatz ihren Anfang nahm, nach und nach sowie allmählich vollzogen hat. Und zum anderen, dass das Ergebnis der bisherigen Veränderung zur Betrachtzeit gültig ist.

Es gibt wenige Belege, in denen Präsensperfekt im übergeordneten Satz einen Sachverhalt mitteilt, der mit dem Beginn der durch den seitTemporalsatz angegebenen Zeitspanne beendet wurde, $d$. h. dass der Sachverhalt im übergeordneten Satz, das zur Betrachtzeit selbst nicht mehr andauert, nur noch einen Gültigkeitsbezug bis zum Betrachtzeitpunkt hat bzw. als resultierender Zustand in diesem vorliegt (28).

(28) Seit es auf der Friesenwiese keinen Flugbetrieb mehr gibt, hat man ihn als über-zählig zur Truppe zurückversetzt. (HHH; 193)

$\Rightarrow$ Seit es auf der Friesenwiese keinen Flugbetrieb mehr gibt, ist er zurückversetzt.

Wenn im übergeordneten Satz eines seit-Temporalsatzgefüges Adverbiale wie zum ersten Mal erscheinen, dann steht der Sachverhalt im übergeordneten Satz im Präteritum in Aoristbedeutung. Dadurch wird implizit zum Ausdruck gebracht, dass sich der Sachverhalt des übergeordneten Satzes zur Betrachtzeit zum ersten Mal vollzieht, was impliziert, dass der Sachverhalt vorher, also seit Beginn des Sachverhalts im Temporalsatz, nicht stattgefunden hat. Ohne eine solche Temporaladverbiale ergäbe das seit-Temporalsatzgefüge je nach Semantik des einzelnen Verbs im übergeordneten Satz entweder keinen Sinn (29') oder erlangt eine gegenteilige Bedeutung, sofern es sich um einen durativen Sachverhalt im übergeordneten Satz handelt. 
(29) Erst nach einer Weile hob sie den Kopf und die Augen, zum ersten Mal, seit Hans bei ihr saß.

(29') *Erst nach einer Weile hob sie den Kopf und Augen, seit Hans bei ihr saß.

Mitunter kann bei den seit-Temporalsatzgefügen der Sachverhalt im übergeordneten Satz zur Betrachtzeit schon beendet sein. Meist wird durch den Kontext ersichtlich, dass der Sachverhalt im übergeordneten Satz nicht mehr andauert und mitunter wider Erwarten durch das sich Ereignen des gegenteiligen Sachverhalts kurz vor der Betrachtzeit beendet wird und somit nicht mehr vorliegt. Dabei wird die Tatsache, dass der Sachverhalt im übergeordneten Satz eventuell schon vor dem Sachverhalt im Temporalsatz galt, bei der Verwendung eines seit-Temporalsatzgefüges etwas abstrahiert, indem lediglich ausgesagt wird, dass der Sachverhalt im übergeordneten Satz zumindest seit Beginn des Sachverhalts im Temporalsatz gilt.

In den solange-Temporalsatzgefügen wird die zeitliche Erstreckung des Sachverhalts im übergeordneten Satz durch den Temporalsatz festgelegt. Dadurch wird zum Ausdruck gebracht, dass der Sachverhalt im übergeordneten Satz die gesamte Zeit des Sachverhalts im Temporalsatz in Anspruch nimmt. Demzufolge besitzen die Sachverhalte der beiden Teilsätze einen gemeinsamen Anfangs- und Endpunkt. In den solange-Temporalsatzgefügen verlaufen die Sachverhalte beider Teilsätze eine Zeitlang parallel und beziehen sich „nicht nur auf den zeitlichen Bereich der eigentlichen Gegenwart, d.h. der aktuellen Sprechsituation bzw. des Redemomentes in der Gegenwart, sondern darüber hinaus auch auf die Zeit danach, also die Zukunft“ (Nespital 1975: 408-409). Daher lassen sich Gegenwarts- und Zukunftsbezug bei solangeTemporalsatzgefügen nicht immer eindeutig voneinander trennen.

Aufgrund ihrer Eigensemantik kommen die solange-Temporalsatzgefüge in allen drei Zeitstufen und als zeitlich nicht-lokalisierte Temporalsatzgefüge vor. Was Zeitstufenbezug und Tempuskombinationen in den solangeTemporalsatzgefügen betrifft, so erscheint bei Gegenwartsbezug in der Regel in beiden Teilsätzen Präsens (30).

(30) Solange ich nüchtern bin, steigert sich die Angst vor dem Auftritt bis zu dem Augenblick, wo ich die Bühne betrete [...]. (BHA; 15)

Darüber hinaus können bei Zukunftsbezug durchaus übereinstimmende Tempusgrammeme in beiden Teilsätzen der solange-Temporalsatzgefüge vorkommen. Üblich ist, bei futurischem Präsens im übergeordneten Satz das Präsens im Temporalsatz zu verwenden (31). Durch das Präsens im solange- 
Temporalsatz bei Zukunftsbezug wird zum Ausdruck gebracht, dass der Sachverhalt im Temporalsatz mitunter schon zur Sprechzeit gilt.

(31) Solange noch die alte europäische Hackordnung gilt, wird es Probleme geben, gewiß. (GGU; 47)

In den solange-Temporalsatzgefügen mit Vergangenheitsbezug erscheint im Deutschen in beiden Teilsätzen meist ein Präteritum in Aoristbedeutung. Dasselbe gilt auch für das Präsensperfekt, das in erster Linie innerhalb wörtlicher Rede vorkommt (32) (vgl. auch Engel 1988:265 und Herweg 1990:300-301). In solchen Fällen steht wenigstens in einem Teilsatz Präteritum, vor allem dann, wenn sein bzw. haben als Vollverb oder ein Modalverb auftreten (33).

(32) Ihr habt euch ein schönes Leben gemacht, solange wir für euch gearbeitet haben. (Beleg aus: Engel 1988: 265)

(33) Vielleicht gibt es so was in Friedrichshain, aber dort bin ich, solange ich Kind war, kaum gewesen. (SRR; 172)

In den solange-Temporalsatzgefügen mit Vergangenheitsbezug erscheint das Präteritum mit Aoristbedeutung im übergeordneten und im Temporalsatz nur dann, wenn das Temporalsatz-gefüge zeitlich nicht-lokalisiert ist und das Präteritum die Wiederholung eines Sachverhalts im übergeordneten Satz wiedergibt (34). In diesen Temporalsatzgefügen steht die völlige Gleichzeitigkeit beider Sachverhalte ohne Angabe ihres Abschlusses im Vordergrund.

(34) Solange die schossen, war Jan für keinen Zuspruch empfänglich, überreizte sich, bediente falsch, vergaß sogar, den Skat zu drücken, und lauschte immer mit einem seiner kleinen wohldurchgebildeten, sinnlich fleischigen Ohren nach draußen, während wir ungeduldig warteten, daß er dem Spielverlauf nachkomme. (GGB; 290)

Bevorzugt wird in den solange-Temporalsatzgefügen mit Vergangenheitsbezug dagegen ein Präteritum mit Imperfektbedeutung in beiden Teilsätzen (35).

(35) Später wuchsen ihr große Haarbüschel auf den Ohren, aber solange sie noch ganz klein war, sah man an vielen Stellen die Haut durch den flockigen Pelz leuchten. (HMW; 60)

Analog zu den solange-Temporalsatzgefügen mit Gegenwartsbezug beziehen sich die Sachverhalte bei den solange-Temporalsatzgefügen mit Vergangenheitsbezug nicht nur auf den Betrachtzeitpunkt in der 
Vergangenheit, von dem aus sie betrachtet werden, sondern jeweils auch auf die darauf folgende Zeit (36).

(36) Bald stand fest, daß sie wohl kaum etwas äußern würde, solange noch eine Nudel auf ihrem Teller läge. (KBI; 206)

Sind die Sachverhalte in beiden Teilsätzen vor einer Betrachtzeit in der Vergangenheit ausgeführt worden und es soll eine kontextuelle relative Vorvergangenheit mitgeteilt werden, dann wird in beiden Teilsätzen jeweils Präteritumperfekt realisiert (37).

(37) Er, Ibrahim, der große Finanzmann des stolzen Sevilla, der Freund und Ratgeber des Emirs, hatte sich zwar von Juden an zu dem Propheten Mohammed bekannt, aber er war nicht als Moslem geboren, sondern als Jude, und dieses Gebäude hier, das Castillo de Castro, hatte seinen Vätern gehört, der Familie Ibn Esra, solange die Moslems in Toledo geharrscht hatten. (FLJ; 14)

\section{Abschließende Bemerkungen}

In dem vorliegenden Beitrag wurden unter Einbeziehung unterschiedlicher sprachlicher Mittel und anhand von standardsprachlichen deutschen Quellentexten aus der Belletristik einerseits der Zeitstufenbezug und das gegenseitige Verhältnis der Tempora, die als Zeitinformatoren gelten, in den während-, al-s, wenn-, seit(dem)- und solange-Temporalsatzgefügen der völligen Gleichzeitigkeit im Deutschen untersucht und der Zeitwert der relativen Tempora, also unter anderem ihre zeitliche Leistung, andererseits überprüft. Darüber hinaus ging es in dem vorliegenden Beitrag um die Kompatibilität der Tempora im übergeordneten Satz und im Adverbialsatz.

Wie bereits in den einleitenden Bemerkungen angedeutet, lassen sich Grenzen zwischen den Temporalsatzgefügen der völligen Gleichzeitigkeit und der partiellen Gleichzeitigkeit nicht immer eindeutig ziehen. Wenn beide Sachverhalte über eine gewisse Duration verfügen, kann man zum einen nicht immer klar entscheiden, ob der eine Sachverhalt nur ein Intervall mit dem anderen gemeinsam hat und ob beide Sachverhalte partiell gleichzeitig verlaufen, oder aber zum anderen, ob beide dieselbe Duration aufweisen und völlig gleichzeitig, also simultan verlaufen. Dabei können die Temporalangaben, welche die Dauer eines der Sachverhaltes limitieren, zur richtigen Einordnung eines Temporalsatzgefüges beitragen. Wenn demzufolge der übergeordnete Satz eine Temporalangabe enthält, dann wird 
darauf hingewiesen, dass der Sachverhalt im übergeordneten Satz nur über eine kürzere Dauer als der Sachverhalt im Temporalsatz verfügt.

\section{QUELLENVERZEICHNIS}

BHA Böll, Heinrich (1992). Ansichten eines Clowns. Köln: Kiepenheuer \&.Witsch Verlag.

BHV Böll, Heinrich (1991). Die verlorene Ehre von Katharina Blum, oder Wie Gewalt entstehen und wohin sie führen kann. Köln: Kiepenheuer \& Witsch Verlag.

FLJ Feuchtwanger, Lion (1999). Die Jüdin von Toledo. Berlin: Aufbau Taschenbuch Verlag.

GGB Grass, Günter (1992). Die Blechtrommel. Darmstadt und Neuwied: Luchterhand Verlag.

GGU Grass, Günter (1992). Unkenrufe. Eine Erzählung. Göttingen: Steidl Verlag.

GJS Gotthelf, Jeremias (1999). Die schwarze Spinne. München: Ullstein Taschenbuchverlag.

HHH Hartung, Hugo (1965). Der Himmel war unten. Frankfurt am Main: Ullstein Taschenbuchverlag.

HMW Haushofer, Marlen (1995). Die Wand. Stuttgart: Klett Verlag.

KBI Kirchhoff, Bodo (1991). Infanta. Frankfurt am Main: Suhrkamp Verlag.

KFV Kafka, Franz (1983). Die Verwandlung. Frankfurt am Main: Fischer Taschenbuch Verlag.

MHR Martin, Hansjörg (1988). Der Rest ist Sterben. Reinbek bei Hamburg: Rowohlt Verlag.

MTB Mann, Thomas (1993). Buddenbrooks. Verfall einer Familie. Frankfurt am Main: Fischer Verlag.

RIS Rodrian, Irene (1992). Strandgrab. Reinbek bei Hamburg: Rowohlt Verlag.

SRR Schneider, Rolf (1975). Die Reise nach Jaroslav. Darmstadt und Neuwied: Luchterhand Verlag.

SPP Süskind, Patrick (1990). Das Parfüm. Die Geschichte eines Mörders. Frankfurt am Main: Gutenberg Verlag.

SVD Schatten, Viola (1991). Dienstag war die Nacht zu kurz. Frankfurt am Main: Fischer Verlag.

ZEIT DIE ZEIT, Wochenzeitung für Politik, Wirtschaft, Handel und Kultur. Hamburg.

ZGC Zwerenz, Gerhard (1977). Zurück in die City - aber nur in die kleine Großstadt neben der großen Großstadt. In: Gerhard Zwerenz, Die Westdeutschen: Erfahrungen, Beschreibungen, Analysen. München: Bertelsmann Verlag.

\section{LITERATURVERZEICHNIS}

Ballweg, J. (1988). Die Semantik der deutschen Tempusformen. Eine indirekte Analyse im Rahmen einer temporal erweiterten Aussagenlogik. Sprache der Gegenwart 70. Düsseldorf: Schwann Verlag.

Engel, U. (1988). Deutsche Grammatik. Heidelberg: Julius Groos Verlag.

Herweg, M. (1990). Zeitaspekte. Bedeutung von Tempus, Aspekt und temporalen Konjunktionen. Wiesbaden: Deutscher Universitätsverlag.

Lutzeier, P. R. (1981). Wahrheitsdefinitorische Überlegungen zur temporalen Lesart der Konjunktion „während“. In: Linguistische Berichte 76. Wiesbaden: Viehweg \& Sohn Verlag. S. 1-24. 
Nespital, H. (1975). Zur syntaktischen Verwendung der Verbalaspekte im Hindu und Urdu. Untersucht am Beispiel temporaler Satzgefüge mit einmaligem Zeitbezug. In: Zeitschrift für Phonetik, Sprachwissenschaft und Kommunikationsforschung. Berlin: AkademieVerlag. Bd. 28. S. 398-431.

Neumann, I. (1972). Temporale Subjunktionen. Syntaktisch-semantische Beziehungen im heutigen Deutsch. Forschungsberichte des Instituts für Deutsche Sprache; Bd. 11. Tübingen: Gunter Narr Verlag.

Pott, H. (1984). Zum Ausdruck der Temporalität. In: Krenn, H., Niemeyer, J., Eberhardt, U. (Hrsg.): Sprache und Text. Akten des 18. Linguistischen Kolloquiums Linz 1983. Bd. I. Tübingen: Lang Verlag. S. 105-117.

Wierzbicka, M. (1999). Das Tempus- und Aspektsystem im Deutschen und Polnischen. Versuch einer integralen Theorie im Rahmen einer temporal erweiterten Aussagenlogik. Sammlung Groos Bd. 70. Heidelberg: Julius Groos Verlag.

Wierzbicka, M. (2004). Zeitbeziehungen in den Temporalsatzgefügen erörtert an den Gegebenheiten der Consecutio Temporum im Deutschen und im Polnischen. München: Iudicium Verlag.

Wierzbicka, M., Schlegel, D. (2008). Sprechzeiten im Diskurs. Zum absoluten und relativen Gebrauch der Tempora in der gesprochenen deutschen Sprache. München: Iudicium Verlag.

Zschunke, S. (1970). Untersuchungen zur Bedeutung und Verwendung der subordinierenden temporalen Konjunktionen in der deutschen Schriftsprache der neueren und neuesten Zeit. Dissertation. Potsdam.

Zschunke, S. (1973). Die subordinierenden temporalen Konjunktionen in der deutschen Schriftsprache der Gegenwart. In: Sbornik Pedagogické Fakulty - Jazyk a Literatura XI. Praha. S. 89-107. 